\title{
Overcoming the Boundary Layer Turbulence at Dome C: Ground-Layer Adaptive Optics versus Tower
}

\author{
T. TRAVOUILLON \\ California Institute of Technology, Pasadena, CA 91125; tonyt@ caltes ch.edu \\ L. JOLISSAINT \\ Observatory of Leiden, Leiden 2333, The Netherlands \\ AND \\ M. C. B. Ashley, J. S. Lawrence, and J. W. V. Storey \\ School of Physics, University of New South Wales, Sydney, NSW 2052, Australia \\ Received 2008 December 15; accepted 2009 April 24; published 2009 May 22
}

\begin{abstract}
The unique atmospheric conditions present at sites such as Dome $\mathrm{C}$ on the Antarctic plateau are very favorable for high spatial resolution astronomy. At Dome $\mathrm{C}$, the majority of the optical turbulence is confined to a 30 to $40 \mathrm{~m}$ thick stable boundary layer that results from the strong temperature inversion created by the heat exchange between the air and the ice-covered ground. To fully realize the potential of the exceptionally calm free atmosphere, this boundary layer must be overcome. In this article we compare the performance of two methods proposed to beat the boundary layer: mounting a telescope on a tower that physically puts it above the turbulent layer, and installing a telescope at ground level with a ground-layer adaptive optics system. A case is also made to combine these two methods to further improve the image quality.
\end{abstract}

\section{INTRODUCTION}

Dome $\mathrm{C}$ is currently the most promising site worldwide for optical/infrared astronomy. Its high altitude, low temperature, and low absolute humidity make it particularly attractive for near-infrared and longer wavelengths, where the sky brightness and transparency are the best of any ground-based site (Lawrence 2004; Walden et al. 2005). For high-resolution observations, however, the site conditions are mixed. Measurements of the turbulence profile responsible for the seeing show that the free atmosphere is exceptionally stable. This stability arises because the Antarctic continent is largely protected from the high-altitude winds and from warm air intrusion by the circumpolar vortex. Such conditions were observed at the South Pole (Marks et al. 1999), a site that is even more centrally located with respect to the vortex. However, the superb free-atmosphere conditions are not the only feature of the Antarctic plateau. The ice-covered ground radiatively cools below the atmospheric temperature, and hence creates a very strong temperature inversion in the air above it. Coupled with near-ground wind shear, this thermal gradient creates a very turbulent ground layer that is severely detrimental to image quality (Gillingham 1991). The height of this turbulent layer has been measured by two independent methods to be $\sim 30 \mathrm{~m}$ above ground level (Lawrence et al. 2004a; Agabi et al. 2006; Trinquet et al. 2008). With an integrated groundlevel seeing well above one arcsec, Dome $\mathrm{C}$ is not competitive with the best midlatitude sites in terms of achievable resolution. That is, unless the effects of the boundary layer, which contributes $\sim 90 \%$ to the total integrated atmospheric turbulence, can be negated. If this can be achieved, then Dome $\mathrm{C}$ appears to be twice as good as the best temperate siteswith a median free-atmosphere seeing of about $0.3^{\prime \prime}$ at $500 \mathrm{~nm}$.

To reach such a goal, two solutions can be proposed. The most intuitive one is to place a telescope on a tower that is taller than the height of the boundary layer. The tower structure must be stiff enough that it resists wind-induced vibrations, and it must be designed to avoid generation of extra turbulence in the telescope beam. The thermal behavior of the tower structure is also critical, as a temperature difference between the tower and the surrounding air could generate convection, creating additional optical turbulence. The optimal height of the tower depends on the percentage of the time that the varying boundary layer is below its top level. More statistical knowledge of the boundary-layer vertical structure is needed to determine the optimum design of such a tower. A concept for a tower-mounted Dome $\mathrm{C}$ telescope has recently been proposed (Saunders et al. 2008). In this design, a very stiff tower is used in conjunction with a tip-tilt secondary mirror to correct for residual tower vibrations. Such a system should achieve an image quality that is close to the free-atmosphere seeing over very wide fields (Saunders et al. 2008). 
The alternative to the tower solution is to use ground-layer adaptive optics (GLAO), an AO mode first proposed by Rigaut (2001) that preferentially corrects the optical turbulence generated by the ground layer. The GLAO principle is relatively simple. As seen from any guide star in the telescope field of view (FOV), the optical beam wave front aberration due to the ground layer looks the same, i.e., the ground layer is highly isoplanatic. This is not true for high-altitude turbulent layers: overlap of the guide stars' optical beams decreases with the layer's altitude, as does the correlation of the guide stars' wave fronts. Therefore, a deformable mirror that is conjugated to the telescope entrance pupil and is driven by the average wave front measured on a set of guide stars distributed across the telescope FOV would correct preferentially the ground layer, leaving the high-altitude layers basically uncorrected. As a consequence, in comparison to a single natural guide star (NGS) or laser guide star (LGS)based AO mode, the correction in GLAO mode extends over a larger FOV, but at the cost of a lower level of performance-as the high altitude turbulence is not compensated. Thus, a GLAO system should achieve a similar resolution to the tower solution, although now the technical challenges are transferred to the telescope optical system (i.e., building a multiple guide star AO system), rather than to the observatory architecture (i.e., building a telescope on top of a 30-40 m tower). In GLAO mode, the best seeing reduction is expected at sites where the largest proportion of the total seeing is in the ground layer. As shown in Table 1, GLAO is a natural choice for Dome C.

In this article, we investigate the performance of a $2.4 \mathrm{~m} \mathrm{di}$ ameter telescope for both $30 \mathrm{~m}$ tower and GLAO options, with the aim of determining the best solution to exploit the excellent seeing conditions at Dome C. A $2.4 \mathrm{~m}$ diameter telescope is chosen as it has been proposed that the next generation optical/ infrared astronomical facility at Dome $\mathrm{C}$ will be of this size (see, e.g., Burton et al. 2005; Saunders et al. 2008). The metrics used to compare the image quality resulting from the two methods are the Strehl ratio, the FWHM (full-width at half maximum) of the corrected point spread function (PSF), and the encircled energy (EE) within a $0.2^{\prime \prime}$ aperture. The simulation is performed using the analytic code PAOLA (Performance of Adaptive Optics for Large Apertures) which models the telescope PSF in both GLAO and seeing-limited modes. The details of the inputs used for the simulations (such as the atmospheric conditions, and the telescope and GLAO characteristics) are presented in $\S 2.1$. Section 3 gives the results of the simulations, in terms of the achieved image quality, firstly for the GLAO ground-level telescope, secondly for the $30 \mathrm{~m}$ tower-mounted telescope, and finally for a tower-mounted telescope equipped with a GLAO system. This last simulation was added to answer a potential development of astrophysical projects at Dome C. It is indeed possible that GLAO is initially chosen for early telescope projects at Dome $\mathrm{C}$ over the construction of a tower due to the lesser demand on the station logistics. If a tower becomes necessary for the following generation of projects then the already existing GLAO system could be used in addition to the tower and further improve the resolution of the telescope.

\section{SIMULATION INPUTS}

\subsection{GLAO System Modeling}

In GLAO, the main contribution to the residual wave-front aberration comes from the so-called angular anisoplanatic error. The correlation between the turbulent phase in the direction of a given science target, $\theta_{s}$, and the turbulent phase in the direction of the guide star, $\theta_{\mathrm{gs}}$, is only partial (the larger the beam's lateral shift, $\left|\theta_{s}-\theta_{\text {gs }}\right| \times h_{\text {layer }}$, the smaller the correlation), therefore there is still some uncorrected turbulent phase at the output of a GLAO system. There are other sources of error: deformable mirror fitting error, wave-front sensor (WFS) aliasing, AO loop time lag, and WFS noise. For a GLAO system, as the FOV or field of correction is rather large ( $\Delta \theta$ is in the range $1^{\prime}-10^{\prime}$ ), angular anisoplanatism is usually the dominant error source (provided that the guide stars are bright enough).

The effect of WFS noise is important for a GLAO system, as it determines the system's sky coverage factor. This sky coverage factor represents the probability that a series of bright guide stars can be found within a given area of sky for an NGS system. If this probability is low, then a laser guide star constellation is required. Our objective here, however, is to determine how well the GLAO solution performs relative to the tower solution in normal conditions, i.e., without considering pathological conditions like photon-starved wave-front sensing regimes. Therefore, we assume that the guide stars are bright enough to

TABLE 1

Summary of the Total, Ground-LAyer, AND FreE-ATMOSPHERE SEEING $\left(\varepsilon_{\mathrm{TOT}}, \varepsilon_{\mathrm{GL}}\right.$, AND $\varepsilon_{\mathrm{FA}}$, RESPECTIVELY) AND THE Isoplanatic Angle $(\theta)$ at Several Major Sites

\begin{tabular}{|c|c|c|c|c|c|}
\hline Site & $\varepsilon_{\mathrm{TOT}}(")$ & $\varepsilon_{\mathrm{GL}}(")$ & $\varepsilon_{\mathrm{FA}}\left({ }^{\prime \prime}\right)$ & $\theta\left({ }^{\prime \prime}\right)$ & Ref. \\
\hline Mauna Kea, Hawaii & 0.74 & 0.52 & 0.45 & 1.9 & Roddier et al. (1990), NAOJ (2003) \\
\hline Cerro Paranal, Chile........... & 0.73 & 0.55 & 0.40 & 1.91 & Murtagh \& Sarazin (1993) \\
\hline La Palma, Canary Isl. . ............ & 0.96 & 0.73 & 0.52 & 1.30 & Vernin et al. (1992) \\
\hline South Pole $\ldots \ldots \ldots \ldots \ldots \ldots \ldots$ & 1.86 & 1.78 & 0.37 & 3.23 & Marks et al. (1999) \\
\hline Dome $\mathrm{C}$ (above $30 \mathrm{~m}) \quad \ldots \ldots \ldots \ldots$ & 0.27 & 0.09 & 0.24 & 5.70 & Lawrence et al. (2004a) \\
\hline
\end{tabular}


neglect WFS noise, and are positioned in a regular pattern, as for an LGS constellation.

We are using an analytic method to compute the GLAOcorrected PSF. This method, described in detail in Jolissaint et al. (2006), relates the residual phase spatial power spectrum to the long-exposure PSF. Basically, in a first approximation, the optical transfer function (OTF, Fourier transform of the PSF) of an $\mathrm{AO}$ system can be written as the product of the telescope OTF and an $\mathrm{AO}$ filter (or AO-OTF): $\mathrm{OTF}_{\text {tot }}=\mathrm{OTF}_{\mathrm{tsc}} \mathrm{OTF}_{\mathrm{AO}}$. The telescope OTF can be calculated analytically using expressions given, for instance, in Schroeder (2000). The AO-OTF is related to the phase structure function $D_{\varphi}$ via $\operatorname{OTF}_{\mathrm{AO}}(\nu) \approx$ $\exp \left[-D_{\varphi}(\lambda \nu) / 2\right]$ (see Jolissaint et al. (2006) for details), where $\nu$ is the angular frequency vector in the focal plane, $\lambda$ is the optical wavelength, and the structure function is defined as the average quadratic difference between the phase at two locations in the pupil plane separated by a given distance, $D_{\varphi}(\mathbf{r})=\left\langle[\varphi(\rho+\mathbf{r})-\varphi(\rho)]^{2}\right\rangle_{\rho}$. Now, the phase structure function is connected to the residual phase spatial power spectrum via $D_{\varphi}(\mathbf{r})=2 \iint[1-\cos (2 \pi \mathbf{f} \cdot \mathbf{r})] \operatorname{PSD}(\mathbf{f}) d^{2} f$ where $\mathbf{f}$ is the spatial frequency in the pupil plane, and as shown in Jolissaint (2008), for the angular anisoplanatic error, we can derive the aniso-servo power spectrum density function:

$$
\begin{aligned}
\operatorname{PSD}_{A S}(\mathbf{f})= & \sum_{n=1}^{N_{l}} \operatorname{PSD}_{\mathrm{atm}}\left(\mathbf{f}, h_{n}\right) \\
& \times \frac{1}{N_{s}^{2}}\left(\sum _ { i = 1 } ^ { N _ { s } } \left[1-2 \sin c\left(\Delta t \mathbf{f} \cdot \mathbf{v}_{n}\right)\right.\right. \\
& \times \cos \left\{2 \pi \mathbf{f} \cdot\left[h_{n}\left(\theta_{i}-\theta\right)+t_{\mathrm{lag}} \mathbf{v}_{n}\right]\right\} \\
& \left.+\sin c^{2}\left(\Delta t \mathbf{f} \cdot \mathbf{v}_{n}\right)\right] \\
& +2 \sum_{i=1}^{N_{s}-1} \sum_{j=i+1}^{N_{s}}\left\{1-\sin c\left(\Delta t \mathbf{f} \cdot \mathbf{v}_{n}\right)\right. \\
& \times\left(\cos \left\{2 \pi \mathbf{f} \cdot\left[h_{n}\left(\theta_{j}-\theta\right)+t_{\mathrm{lag}} \mathbf{v}_{n}\right]\right\}\right. \\
& \left.+\cos \left\{2 \pi \mathbf{f} \cdot\left[h_{n}\left(\theta_{i}-\theta\right)+t_{\mathrm{lag}} \mathbf{v}_{n}\right]\right\}\right) \\
& \left.\left.+\sin c^{2}\left(\Delta t \mathbf{f} \cdot \mathbf{v}_{n}\right) \cos \left[2 \pi h_{n} \mathbf{f} \cdot\left(\theta_{j}-\theta_{i}\right)\right]\right\}\right)
\end{aligned}
$$

where

$\operatorname{PSD}_{\text {atm }}\left(\mathbf{f}, h_{n}\right)$ is the spatial power spectrum of the turbulent phase at the altitude $h_{n}$, given by the Von Karman formula $\mathrm{PSD}_{\mathrm{atm}}\left(\mathbf{f}, h_{n}\right)=0.0229 r_{0}\left(h_{n}\right)^{-5 / 3}\left(f^{2}+1 / L_{0}^{2}\right)^{-11 / 6}, \quad$ where the so-called Fried parameter (Fried 1966), $r_{0}\left(h_{n}\right)$, is a measure of the lateral spatial coherence of the phase aberration associated with the turbulent layer at altitude $h_{n}$, and $L_{0}$ is the outer scale of the turbulent flow;

$N_{l}$ is the number of turbulent layers and $N_{s}$ is the number of guide stars;

$\Delta t$ is the WFS integration time; $\mathbf{v}_{n}$ is the wind velocity vector inside the layer at altitude $h_{n}$ (assuming Taylor's hypothesis);

$\theta_{i}$ is the angular position vector of the $i$-th guide star in the sky;

$\theta$ is the angular position vector of the science object (where you want the PSF to be calculated); and

$t_{\text {lag }}$ is the AO loop time lag, i.e., the time lost reading the WFS and computing the deformable mirror command, or the delay between the middle of the WFS exposure and the actual application of the deformable mirror correction.

Equation 1 includes the servo-lag error, as it is correlated with angular anisoplanatism. We use it with typical values for the WFS integration time and AO time lag, although this error is typically much smaller than the angular anisoplanatic error.

The procedure to compute the long-exposure PSF is the following: (1) compute the aniso-servo PSD with equation 1, using as inputs the layer altitudes, the $r_{0}$ per layer, the wind velocity vertical profile, and the guide star locations; (2) compute the phase structure function by numerical integration of the structure function integral; (3) compute the AO-OTF with the exponential of the structure function; (4) compute the telescope's OTF; (5) compute the overall OTF (OTFs product); and (6) compute the PSF by taking the inverse Fourier transform of the OTF (using the FFT algorithm).

To obtain the seeing-limited (uncorrected) PSF, there is no need to use the power spectrum approach, as an analytical expression-including the outer scale-exists for the turbulent phase structure function (Tatarski 1961):

$$
\begin{aligned}
D_{\varphi}(\mathbf{r})= & 0.171661\left(L_{0} / r_{0}\right) \\
& \times\left[1.005635-\left(2 \pi r / L_{0}\right)^{5 / 6} K_{5 / 6}\left(2 \pi r / L_{0}\right)\right]
\end{aligned}
$$

For the seeing-limited case, we simply use this expression to compute the OTF filter associated with the turbulence profile, and then follow the same steps as for the GLAO PSF calculation.

Using this procedure, a long-exposure AO corrected or seeing-limited PSF is obtained in less than a second. This algorithm has been coded into the IDL-based modeling code, PAOLA.

\subsection{GLAO System Parameters}

The parameters of the simulated GLAO system used here are similar to previous publications using PAOLA. We will explore the performance of a GLAO system on a $2.4 \mathrm{~m}$ telescope with a secondary obscuration of $0.56 \mathrm{~m}$ operating in the $V, J, H$ and $K$ bands. We use a wave front sensor integration time of $1 \mathrm{~ms}$ and a control loop time lag of $0.7 \mathrm{~ms}$, values typical for current $\mathrm{AO}$ systems. The actuator pitch has been chosen to be equal to the average value of the Fried parameter $r_{0}$ of the site. We use a value for the outerscale of turbulence, $L_{0}$, of $20 \mathrm{~m}$. Recent results indicate that $L_{0}$ at Dome $\mathrm{C}$ may have a median value as 
TABLE 2

Median Turbulence and Wind Speed Profiles Obtained from the MASS + SODAR and the Microthermals. Microthermal and Wind Speed Profiles were Interpolated to Match the MASS Resolution. Heights are Given as the Center of the Resolution Bin

\begin{tabular}{|c|c|c|c|c|c|c|c|c|}
\hline Height & $15 \mathrm{~m}$ & $220 \mathrm{~m}$ & $500 \mathrm{~m}$ & $1 \mathrm{~km}$ & $2 \mathrm{~km}$ & $4 \mathrm{~km}$ & $8 \mathrm{~km}$ & $16 \mathrm{~km}$ \\
\hline MASS + SODAR $\left[\times 10^{-14} \mathrm{~m}^{1 / 3}\right]$ & - & 0.89 & 2.49 & 0.11 & 1.1 & 1.59 & 1.25 & 0.24 \\
\hline Microthermals $\left[\times 10^{-14} \mathrm{~m}^{1 / 3}\right]$ & 107 & 7.32 & 0.23 & 0.36 & 0.68 & 1.72 & 1.67 & 0.42 \\
\hline Wind speed $\left[\mathrm{m} \mathrm{s}^{-1}\right] \ldots \ldots \ldots$ & 2.7 & 7.15 & 7.45 & 8.13 & 9.8 & 14.6 & 27.05 & 35.4 \\
\hline
\end{tabular}

low as $7.5 \mathrm{~m}$ (Ziad et al. 2008). The value of $L_{0}$, however, has little influence in the case of a small telescope such as the one analyzed here. Three guide stars are the minimum to allow for a relatively good sampling of the low-altitude layers. Our guide
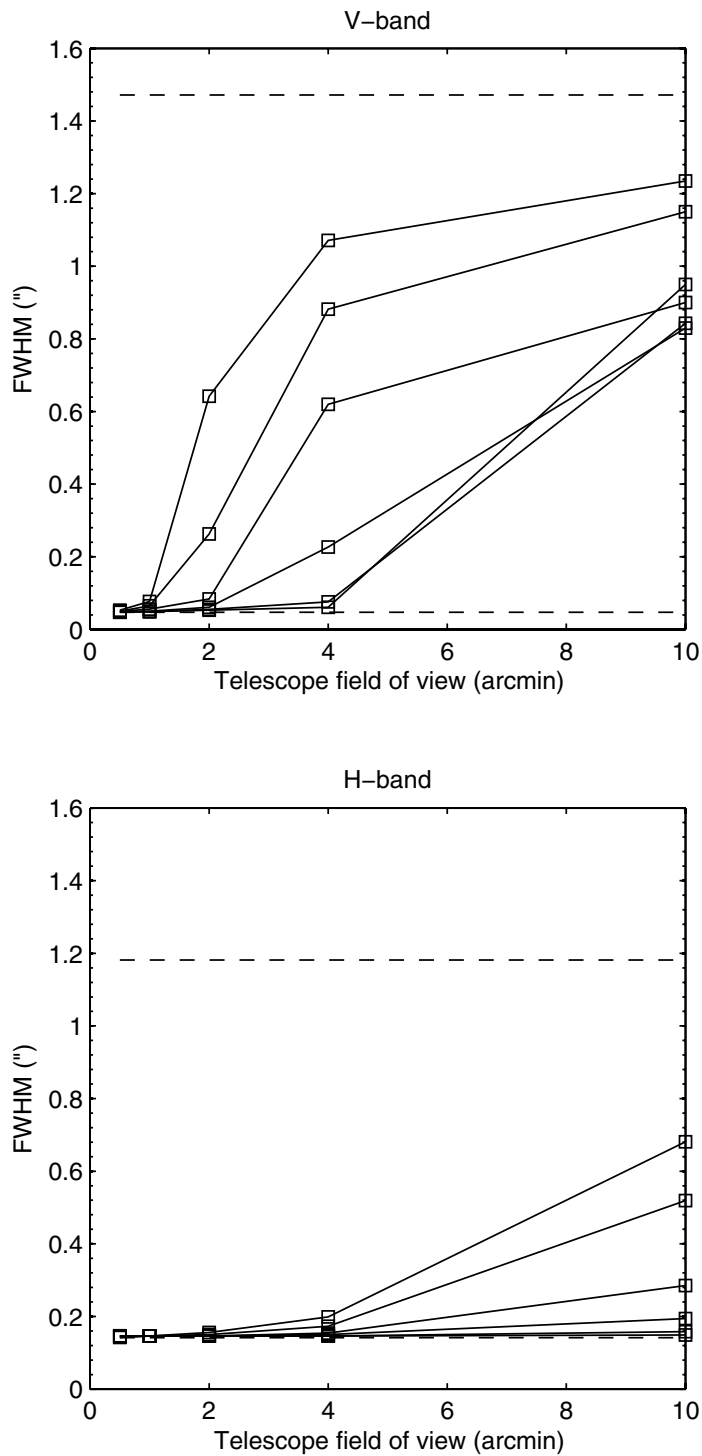

stars are placed at the tips of an equilateral triangle, and we vary the radius of this triangle from $0.5^{\prime}$ to $10^{\prime}$. Note that the larger the number of guide stars, the better the GLAO system performance, and the better the stability of the PSF metrics across
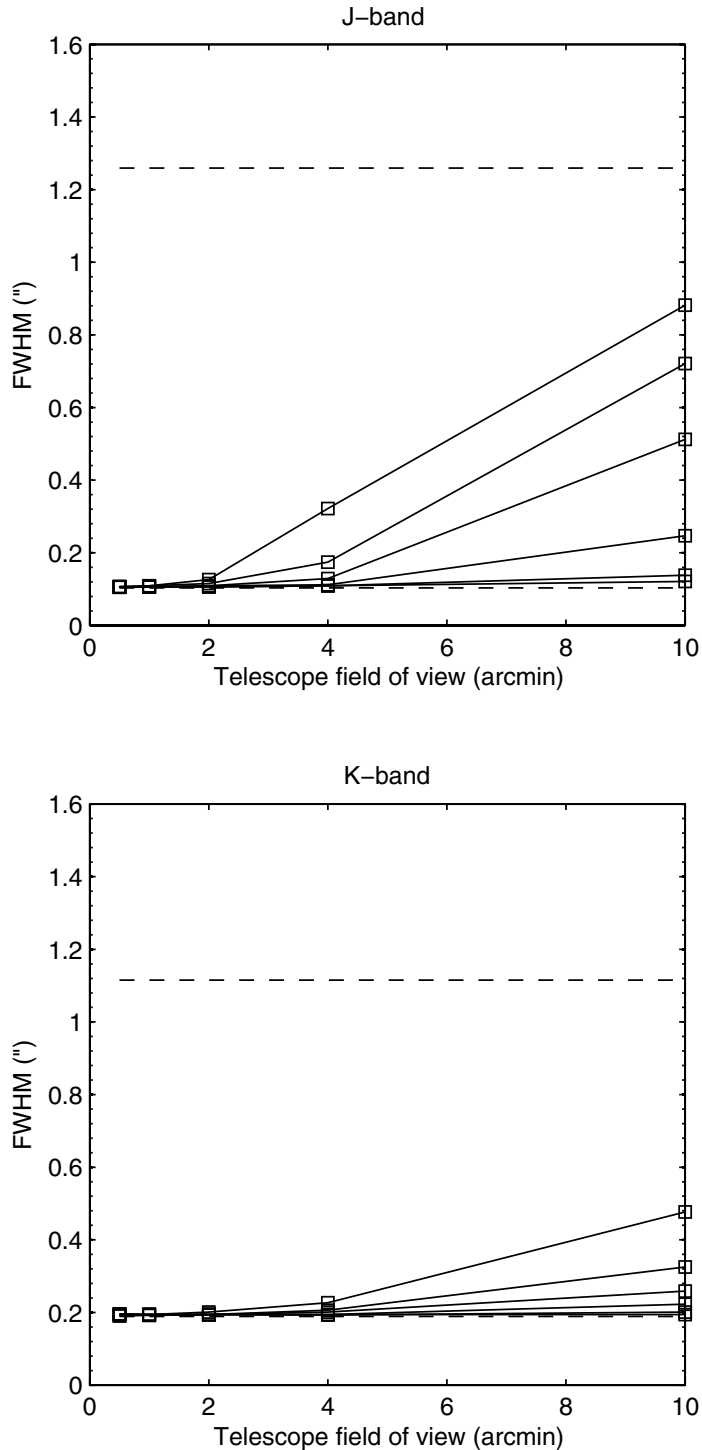

FIG. 1.-GLAO-corrected FWHM as a function of telescope FOV for observations at $V, J, H$, and $K$ wavebands. Each solid line corresponds, from bottom to top respectively, to the image quality at the center of the field to the image quality at the edge of the field in $20 \%$ steps. The upper dashed line corresponds to the natural seeing at this wavelength and the lower dashed line to the diffraction limit. 
the FOV, as shown in Jolissaint (2008). About 10-12 GS arranged in a circle would be needed to give a performance close to the ideal case described by Tokovinin (2004), where the wave front is perfectly known all along the circle defining the GLAO FOV, and not only on a few GS. Now, implementing such a large number of GS is currently not realistic in such a harsh environment as Dome $\mathrm{C}$, and for this very reason, we consider in our study only three GS, which is a minimum, but a good start to get a significant improvement with respect to the seeinglimited case, while minimizing the complexity of WFS design and technical implementation at Dome C.
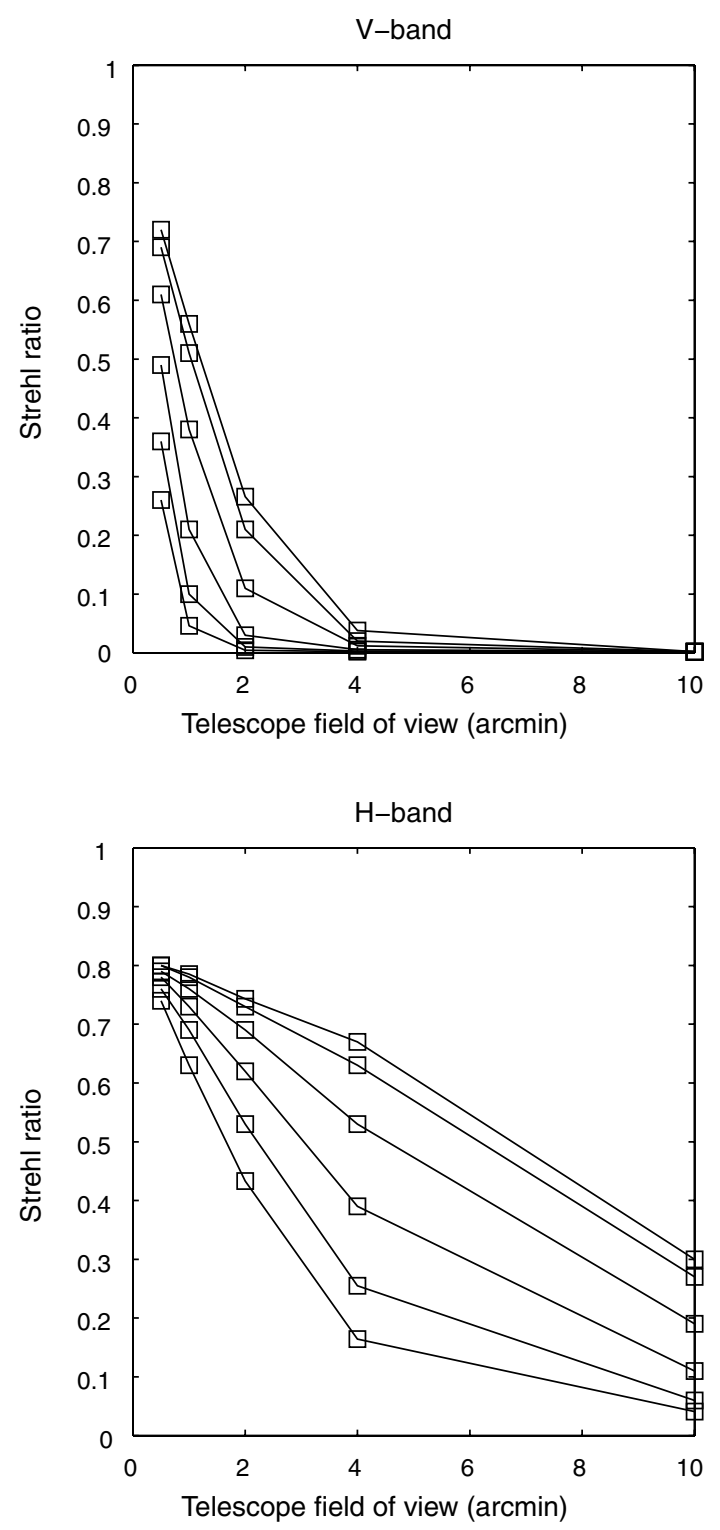

\subsection{Turbulence Profiles}

For the first part of the simulation (GLAO from the ground), the median turbulence and wind speed profiles are used. Ideally, simulations should be performed on individual profiles and the statistical averaging carried out at the end. Unfortunately this cannot be achieved for the ground-based GLAO system as we do not have complete profiles from the ground up. For the altitude range $30 \mathrm{~m}$ to $24 \mathrm{~km}$, we use a median profile of the refractive index structure constant, $C_{n}^{2}$, from a combination of the SODAR and MASS instruments described in Lawrence et al. (2004b). These instruments give $C_{n}^{2}$ in seven range
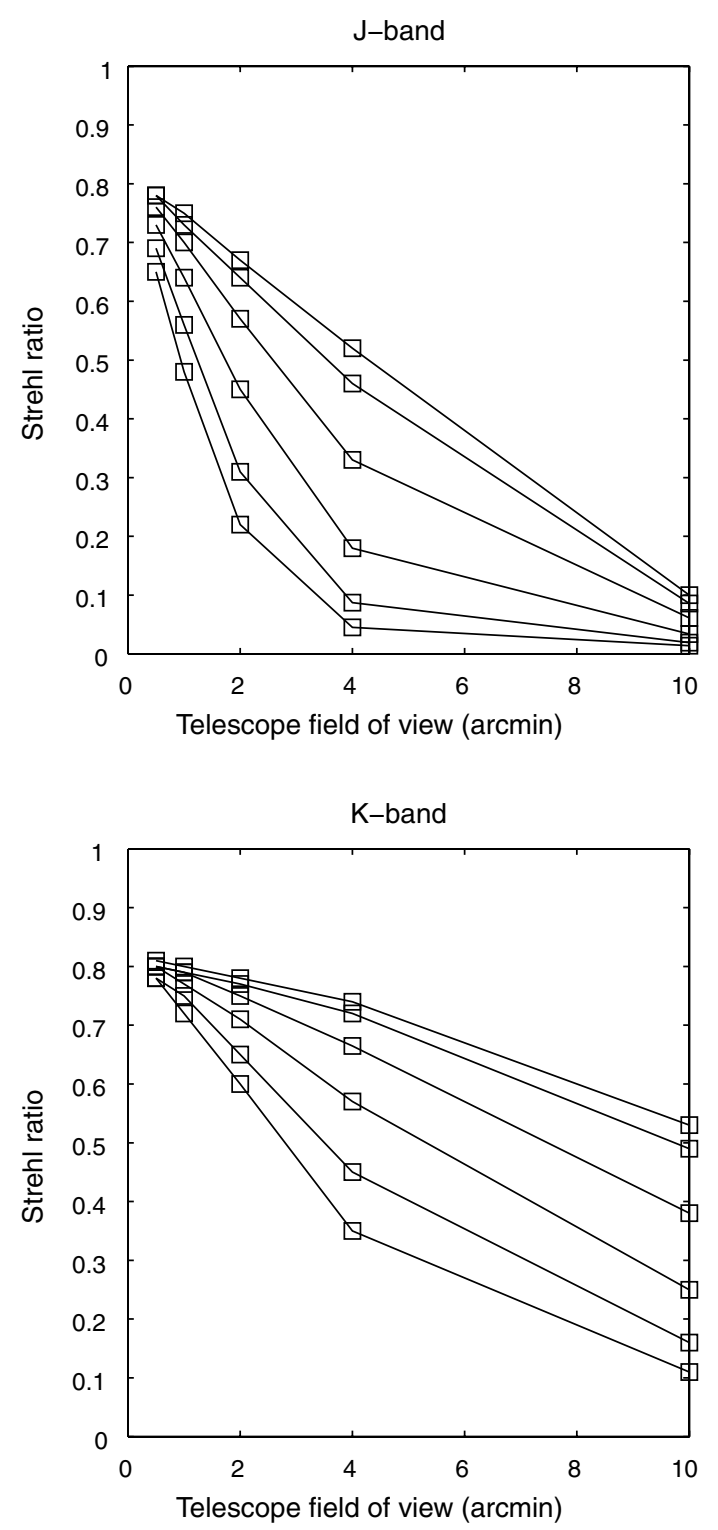

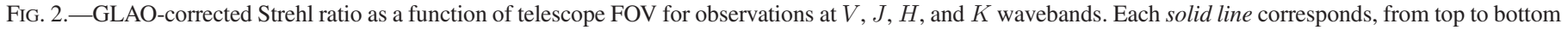
respectively, to the image quality at the center of the field to the image quality at the edge of the field in $20 \%$ steps. 
bins, obtained during a six-week period from 2004 March to 2004 May (Lawrence et al. 2004a). For the altitude range from $7 \mathrm{~m}$ to $30 \mathrm{~m}$, we use the average turbulence recorded by microthermal balloons presented by Agabi et al. (2006), obtained from 16 balloon flights during 2005. It is important to stress that this reconstructed profile, shown in Table 2, is therefore not an ideal representation of the nighttime conditions at Dome $\mathrm{C}$ but is still the most statistically complete that is so far available. Indeed, the data taken by Lawrence et al. may be limited to the fall season, but they contain a large statistical sample of this season (2670 profiles). The Agabi et al. profiles do cover all nighttime seasons but at the cost of a lower statistical sample.

The free atmosphere above Antarctic sites has also been studied using an ECMWF (European Center for Medium Range Forecasts) reanalysis (Hagelin et al. 2008). Although a quantitative estimate of the free-atmosphere seeing is not derived, this analysis provides a sound theoretical explanation for the superior seeing above Antarctic sites such as Dome C.

Having such a constant ground-layer turbulence is actually a good assumption since while the boundary layer height may vary, the integrated turbulence within it is very constant, as shown at the South Pole (see Travouillon et al. 2003). Additionally, the variations of the height of the boundary layer are unlikely to be a detriment to the quality of the GLAO simulation since the conjugation of the deformable mirror is insensitive to variations of a few tens of meters (Travouillon et al. 2004). For all simulations, the wind speed profile used is modeled as a Gaussian distribution scaled to coincide with the median coherence time for the site.

In the study of the image quality achieved by the towermounted telescope, only the turbulence and wind speed profiles from above $30 \mathrm{~m}$ are necessary. We use both the SODAR +
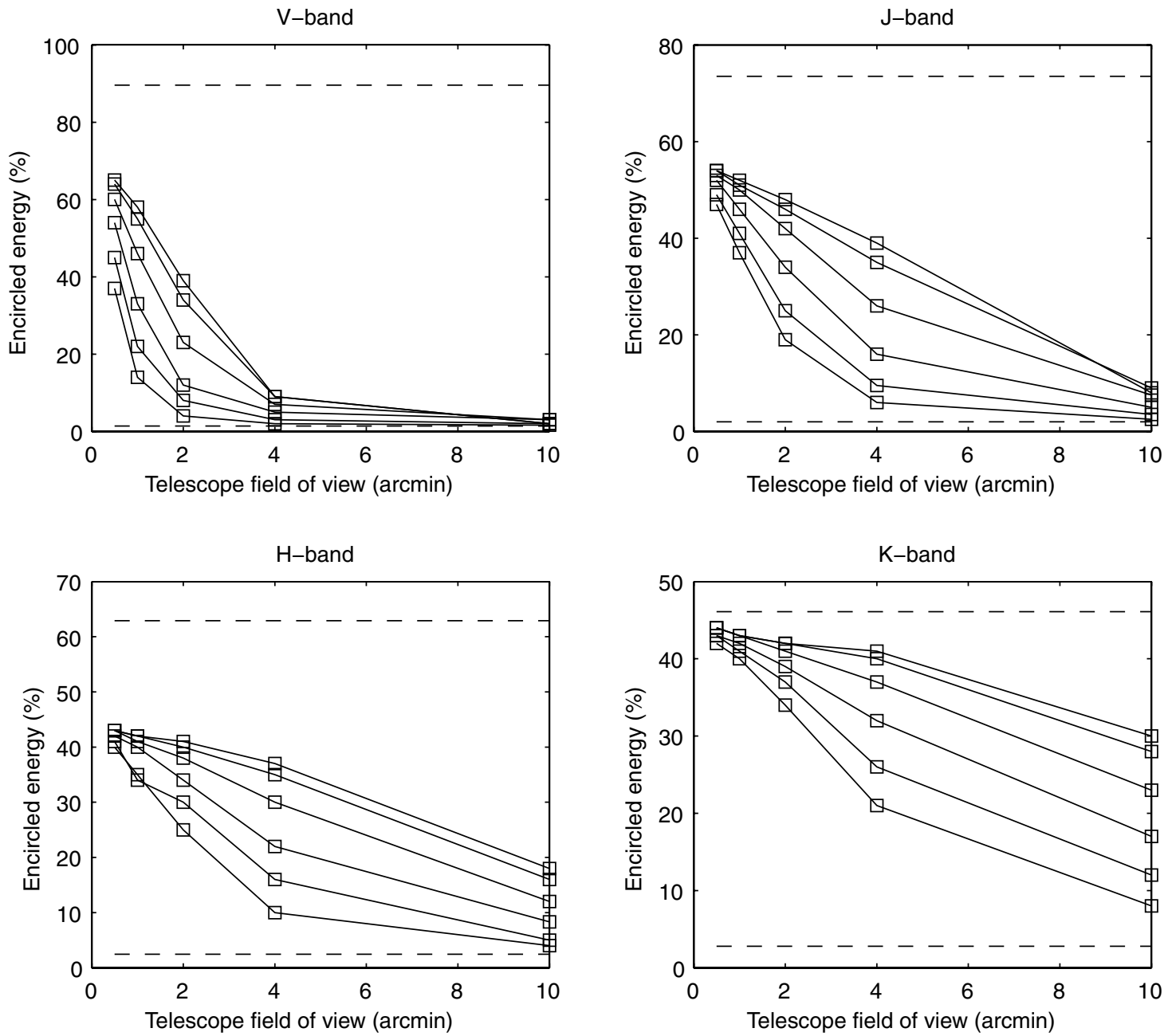

FIG. 3.-GLAO-corrected encircled energy within $0.2^{\prime \prime}$ as a function of telescope FOV for observations at $V, J, H$, and $K$ wavebands. Each solid line corresponds, from top to bottom respectively, to the image quality at the center of the field to the image quality at the edge of the field in $20 \%$ steps. The upper dashed line corresponds to the theoretical limit assuming a $100 \%$ Strehl and the lower line to seeing-limited case. 
MASS profiles and the microthermal profiles, the median from both data sets giving a seeing (at $500 \mathrm{~nm}$ ) of $0.27^{\prime \prime}$ and $0.36^{\prime \prime}$ respectively, when integrated from $30 \mathrm{~m}$ above ground level. It is because of the large difference between the two values $(25 \%)$ that both are used in the simulation. The profiles are shown in Table 2.

In the third part of the simulation, which considers the performance of the same GLAO system based on the $30 \mathrm{~m}$ tower, we have elected to run the analysis on individual SODAR/ MASS profiles. The motivation for this choice is that such simulation will be able to take into account and correct for the variations of height of the boundary layer between the individual profiles. Indeed, the GLAO system will be able to remove the turbulence when the boundary layer is above the nominal $30 \mathrm{~m}$ height of the tower. This analysis is therefore far less valuable on median profiles.

\section{RESULTS}

\subsection{GLAO Results}

The performance of the $2.4 \mathrm{~m}$ ground-based telescope fitted with a GLAO system is largely a compromise between resolution improvement and FOV. As mentioned earlier, the advantage of GLAO is that it offers a correction over a wide FOV. It is therefore logical to study over which FOV a given image quality is achieved. Figures 1, 2 and 3 show the simulation results in units of image FWHM, Strehl ratio, and encircled energy within $0.2^{\prime \prime}$ respectively, as a function of telescope FOV. For all four wavebands, the FOV was varied from $0.5^{\prime}$ to $10^{\prime}$, and three laser guide stars were spread to the edge of the field $\left(120^{\circ}\right.$ from each other). The performance were calculated for science objects located at different radial distances from the center of the field ranging from $0 \%$ (center of the field) to $100 \%$ (edge of the field) in $20 \%$ steps. The relative location of the three LGS and the studied locations of the science object can be seen in Figure 4.

The results show that in $H$ and $K$ bands, the telescope can achieve close to the diffraction limit for fields up to $4^{\prime}$. For larger fields, the image quality is still vastly improved compared to the natural seeing. An improvement over the resolution obtained at the best temperate sites, however, is only obtained close to the center of the field. The location on the field has a more dramatic effect on Strehl ratio. For a 4 ' field in $H$ band the Strehl ratio quickly drops to 0.15 (from about 0.7 in the center). In $J$ band, reasonable GLAO correction is obtained out to $4^{\prime}$, apart from the outer $20 \%$ of the field. In the visible, however, the technique is only beneficial for small fields under $1^{\prime}$. The results of the encircled energy follow closely the results of the Strehl ratios with stronger benefits for large fields in the $K$-band. Indeed, for a field up to $4^{\prime}$, the performance over the entire field is better than half of the theoretical limit of the PSF. It is worth noting that for a $10^{\prime} \mathrm{FOV}$, the performance is better for an off-axis location than on-axis is an indication that the quality of the correction, with

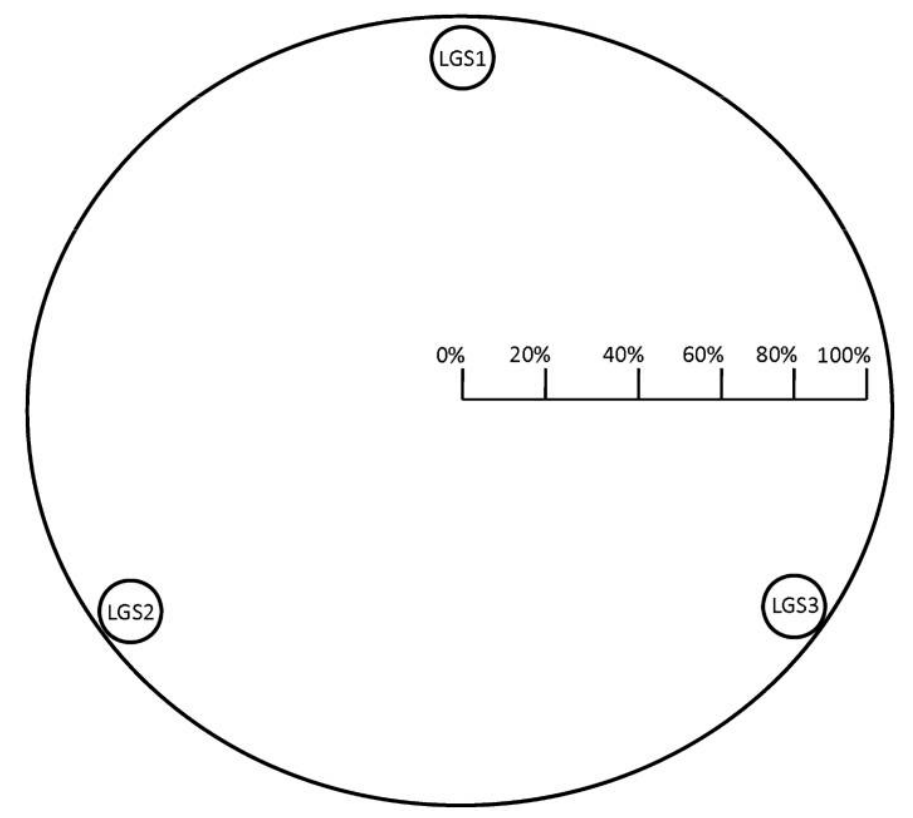

FIG. 4.-Graphical depiction of the relative locations of the three LGSs and science object (which is moved from the center to the edge of the field in $20 \%$ increments).

only three guide stars, varies strongly within the field, and improves in particular when one gets closer to a GS. This argument is in favor of implementing the largest possible number of GS, considering technical and costs limitations. As simulations results shown in Jolissaint (2008) seem to indicate, implementing five rather than three GS would already significantly increase the GLAO system performance over the FOV, toward the ideal case described by Tokovinin (2004).

Our results are comparable with the simulations done by Le Roux et al. (2008) using CAOS. Using relatively similar atmospheric input and telescope size $(2 \mathrm{~m})$, they show that in the $J$-band the Strehl drops from 0.55 to 0.23 when going to $2^{\prime}$ off axis. In our simulation the Strehl drops from 0.78 to 0.21 . For larger fields, however, our Strehls drop faster as we only use three guide stars against four in Le Roux's simulations. Additional differences between the two simulations may be attributed to the difference of turbulence profiles, especially in the ground layer where the correction is the most important. This stresses the importance of using accurate and high-resolution profiles, which is difficult when using average conditions.

\subsection{Tower Results}

The second proposed solution to avoid the strongly turbulent boundary layer is to simply place the telescope above the top of the layer. Current estimates of this height are 30-40 m. Choosing $30 \mathrm{~m}$ for the simulation here is a convenient height considering our turbulence data set, and it has also been shown to be a realistic height for a metallic structure of the kind proposed by Saunders et al. (2008), based on the design of Hammerschlag 
(2006). Ignoring engineering and other considerations, the performance of the same telescope on such a $30 \mathrm{~m}$ tower can be directly compared with the GLAO option from the ground in terms of achieved image quality.
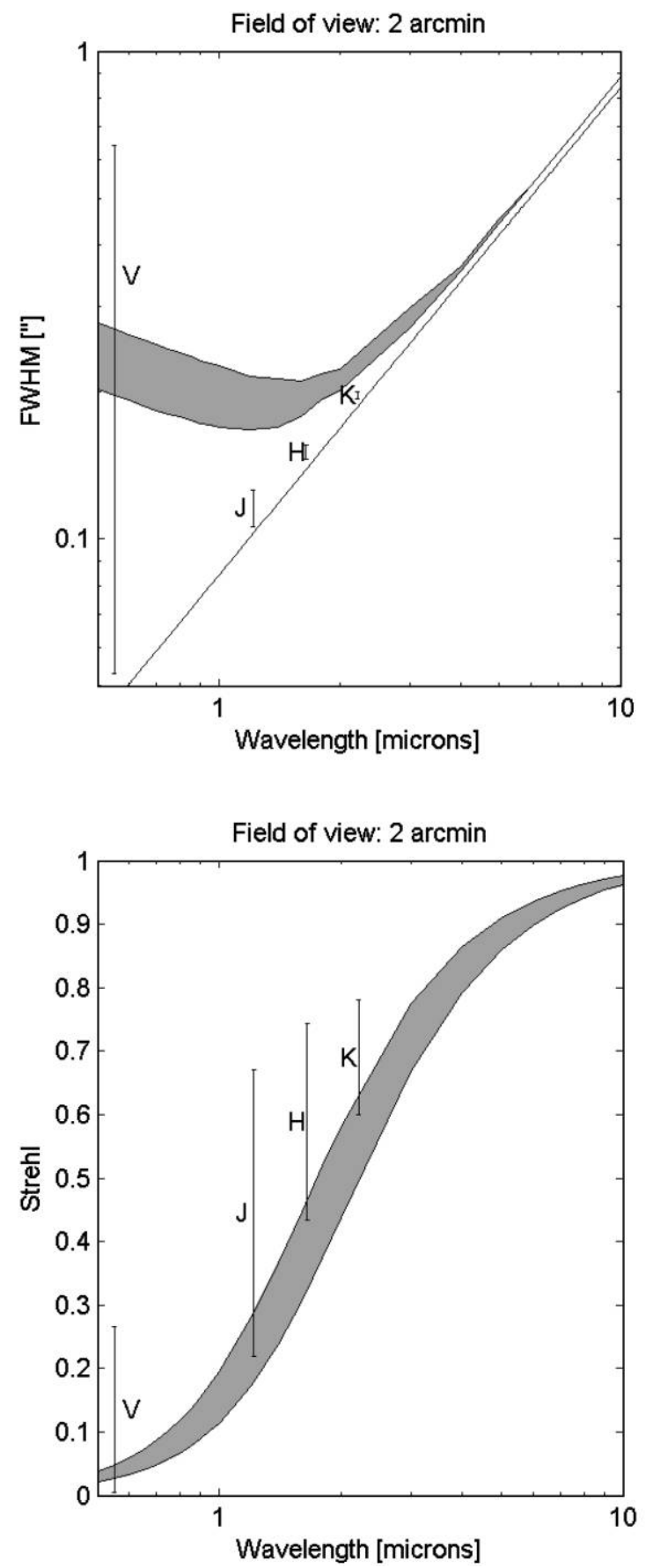

In seeing-limited mode, the performance of the telescope is purely a function of wavelength. Here again, PAOLA is used to simulate the image quality of telescope. Figure 5 shows the FWHM image size and Strehl ratio, as a function of wavelength,
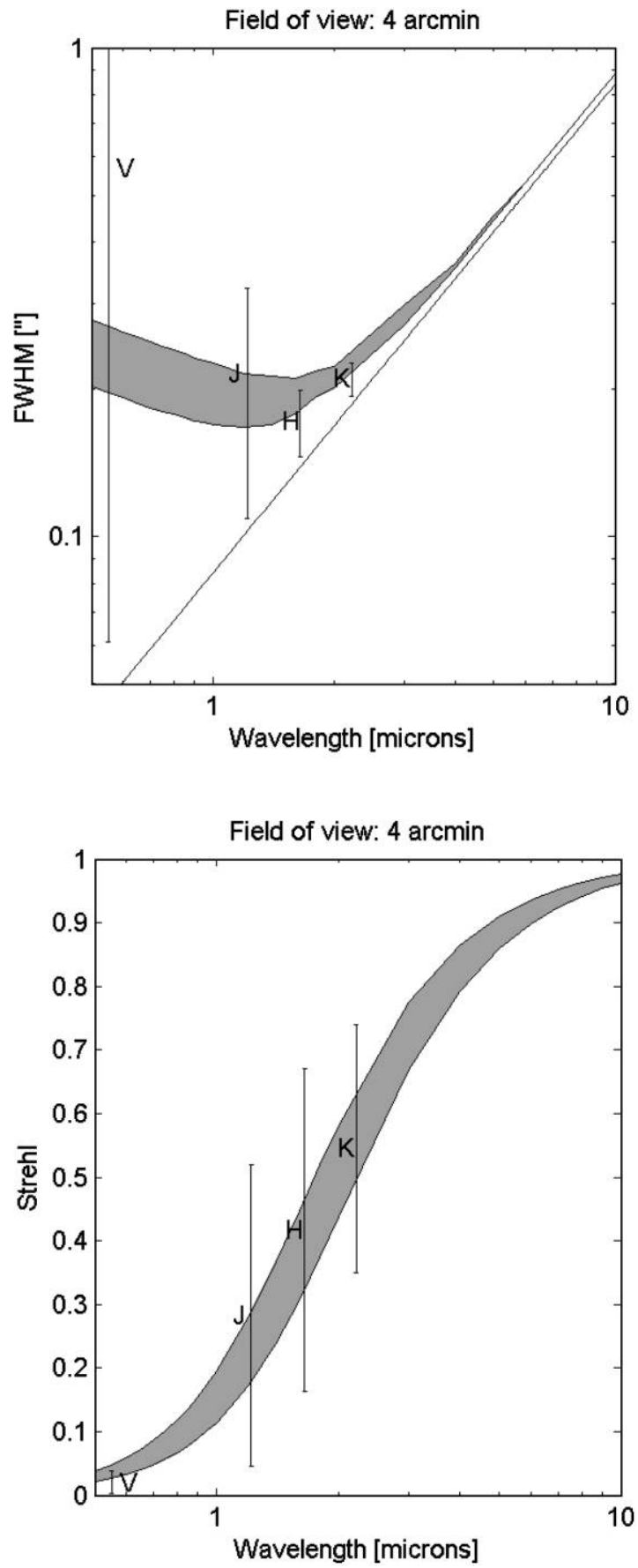

FIG. 5.-Image quality FWHM (top row) and Strehl ratio (bottom row) for a $2.4 \mathrm{~m}$ Dome C telescope mounted on a $30 \mathrm{~m}$ tower as a function of wavelength (gray area) using the SODAR + MASS (lower bound) and the microthermal (upper bound) data sets. The diagonal line in the top row corresponds to the diffraction limit. Overplotted as vertical bars is the performance for a ground-level telescope with a GLAO system for a 2' (left column) and 4' (right column) FOV at $V, J, H$, and $K$ wavebands. Each vertical line spans the performance of the GLAO system from the center of the field (lower end of the line) to the edge of the field (upper end of the line). The tower is assumed to be infinitely stiff. 
using results from the two data sets: the lower bound is simulated using the SODAR + MASS results and the upper bound using the microthermals. To aid the comparison, the previous GLAO results are overplotted on the same figure using FOV of $2^{\prime}$ and $4^{\prime}$. It is important to note that this simulation assumes that the effects of tower vibrations have been removed with a tip-tilt correction. In other words, we assume a perfectly stiff tower.

Apart from $V$ band, the ground-based GLAO system is shown to outperform the tower for the smaller 2 field. For such a small field the AO system effectively removes more than the turbulence in the lowest $30 \mathrm{~m}$, and the tightly packed guide stars correct the entire field properly. The largest gain of the groundbased GLAO system compared to the tower-mounted telescope occurs for wavelengths around $J$ band. For wavelengths at $K$ band and longer, both systems achieve close to the diffraction limit of the telescope.

For fields larger than $4^{\prime}$, the choice of the tower-mounted telescope becomes more appealing since only for longer wavelengths can the GLAO system obtain the same resolution. It should be noted that the modeled resolution obtained for the tower-mounted telescope is derived purely from the integrated seeing at the telescope altitude (i.e., $\sim 30 \mathrm{~m}$ ). There is some discrepancy between the values obtained for the MASS + SODAR data and the microthermal data - profiles from the microthermal data set show some residual boundary-layer turbulence above $30 \mathrm{~m}$, but are in agreement with the MASS + SODAR data in terms of the free-atmosphere seeing (i.e., $\sim 0.3^{\prime \prime}$ ) for heights greater than $\sim 40-50 \mathrm{~m}$.

It is known that wind shake of the tower structure will contribute to image degradation, and that a guiding system will be required to remove this. It was shown by Saunders et al. (2008) that for a $2.4 \mathrm{~m}$ tower-mounted Dome $\mathrm{C}$ telescope, a tip-tilt secondary mirror can effectively correct such wind-induced image degradation. Additionally, this guiding system gives significant adaptive improvement that can correct for residual boundary layer turbulence above the telescope structure. With such a system, the expected image quality is $\sim 0.3^{\prime \prime}$ FWHM (i.e., essentially the free-atmosphere seeing) for wavelengths over the range $0.7-2.5 \mu \mathrm{m}$. This image quality is constant over very wide fields (at least up to $40^{\prime}$ in the visible).

\subsection{GLAO on the Tower}

The previous sections have demonstrated that the groundbased GLAO system can achieve a higher spatial resolution than the tower-mounted telescope, but only for relatively small fields. In order to increase the corrected FOV, we may consider using the two methods in conjunction, i.e., by placing the telescope on a $30 \mathrm{~m}$ tower and fitting it with a GLAO system. This option is mainly a practical consideration as both a GLAO system and a tower may be considered individually by different groups. Depending on the logistical realities on the ground, a GLAO system may see light first and then be followed by
TABLE 3

Statistical Summary of GLAO Performance Averaged OVER THE FIELD ON A TOWER-MOUNTED 2.4 M TELESCOPE WITH A GLAO SySTEM AT DOME C IN THE $V$-BAND FOR A FOV OF 10'. ThE DifFRACTION LIMIT AT THIS WAVELENGTH IS $0.05^{\prime \prime}$

\begin{tabular}{llcccc}
\hline \hline & & Mean & $50 \%$ & $25 \%$ & $75 \%$ \\
\hline Uncorrected FWHM (") & $\ldots .$. & 0.28 & 0.27 & 0.18 & 0.34 \\
Corrected FWHM (") & $\ldots \ldots .$. & 0.19 & 0.17 & 0.12 & 0.25 \\
\hline
\end{tabular}

the construction of a tower. In this case, it is worth studying the benefits of combining the two systems.

The first consideration we need to make concerns the wavelength coverage where GLAO + tower is beneficial. Since the seeing conditions at this elevation are very good, it is easy to see that for a $2.4 \mathrm{~m}$ class telescope, the longer infrared wavelengths will be diffraction limited, even without AO. Indeed, the MASS + SODAR data show that at $K$ band a $2.4 \mathrm{~m}$ telescope will be diffraction limited $89 \%$ of the time. At $H$ and $J$ bands, the diffraction limit is achieved for $68 \%$ and $43 \%$ of the time, respectively. It is only in the $I$ band that diffraction-limited conditions becomes rare enough (17\%) to justify the use of GLAO. At $V$ band the telescope is never diffraction limited with natural seeing. We will therefore focus on the performance improvement in the $V$ and $I$ bands. Additionally, the simulation is run on each of the 2670 SODAR/MASS profiles. This allows us to obtain more realistic results than when comparing median conditions, due to both the variations of the boundary layer height and the realistic nature of the individual profiles.

The $V$-band simulation results are summarized in Table 3 and Figure 6. Because of the stability of the boundary layer

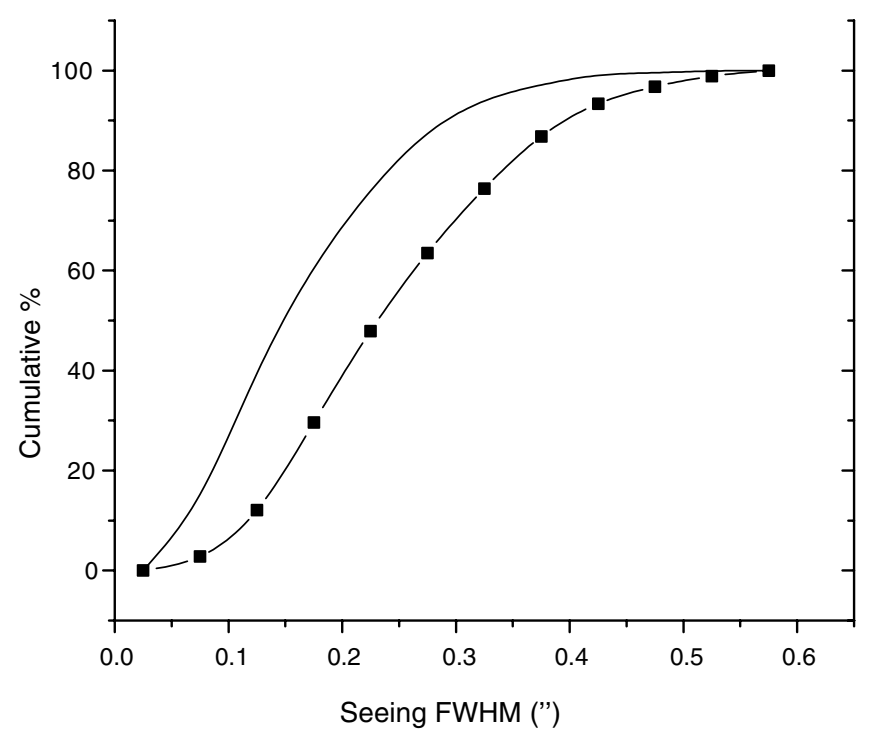

FIG. 6.-Cumulative distribution of the natural (dots) and corrected seeing (line) over a field of $10^{\prime}$ (correction averaged over the field) in the $V$ band for a tower-mounted $2.4 \mathrm{~m}$ Dome C telescope with a GLAO system. 

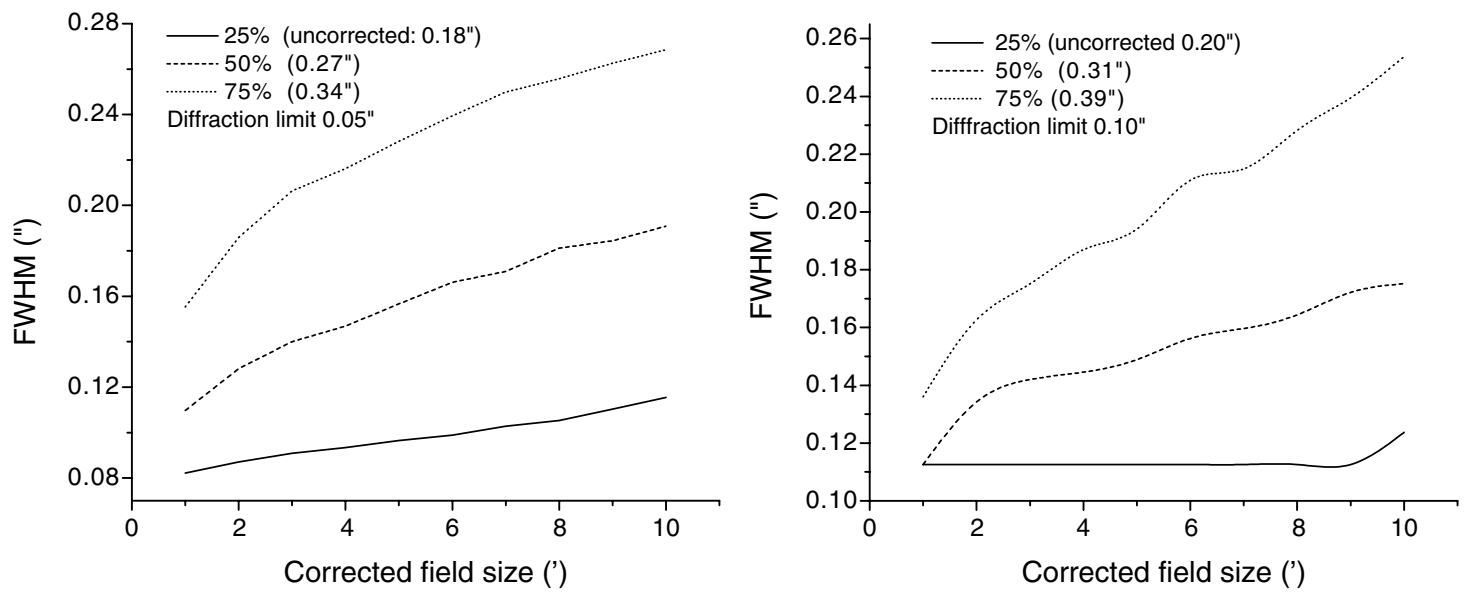

FIG. 7.-Corrected seeing as a function of field size for the best $25 \%, 50 \%$ and $75 \%$ profiles in $V$ band (left plot) and $I$ band (right plot), for a tower-mounted $2.4 \mathrm{~m}$ Dome C telescope with a GLAO system.

we find that a high level of correction can be achieved for very wide FOVs. The correction as a function of FOV is illustrated in Figure 7 for $V$ and $I$ band. On these graphs are shown the 25, 50 , and 75 percentiles of the corrected FWHM as a function of field size. This correction is averaged over the FOV. For the best $25 \%$ conditions in $I$-band, the system reaches diffractionlimited resolution almost out to a $10^{\prime} \mathrm{FOV}$; for the median conditions at this wavelength, diffraction limit is reached over a $1^{\prime}$ field. In the $V$ band $0.1^{\prime \prime}$ resolution is reached out to a $10^{\prime}$
FOV for the $25 \%$ best conditions. For median conditions, there is a large variation of resolution across the field-but in both $V$ and $I$ bands, the FWHM is less than $0.2^{\prime \prime}$ out to at least $10^{\prime}$.

While the statistical results use all 2670 profiles, it is interesting to take a qualitative look at the temporal evolution of the GLAO performance. Figure 8 shows two time series of the natural and corrected seeing in the $V$-band. The left-hand graph, corresponding to the data of 2004 April 1, shows that the level

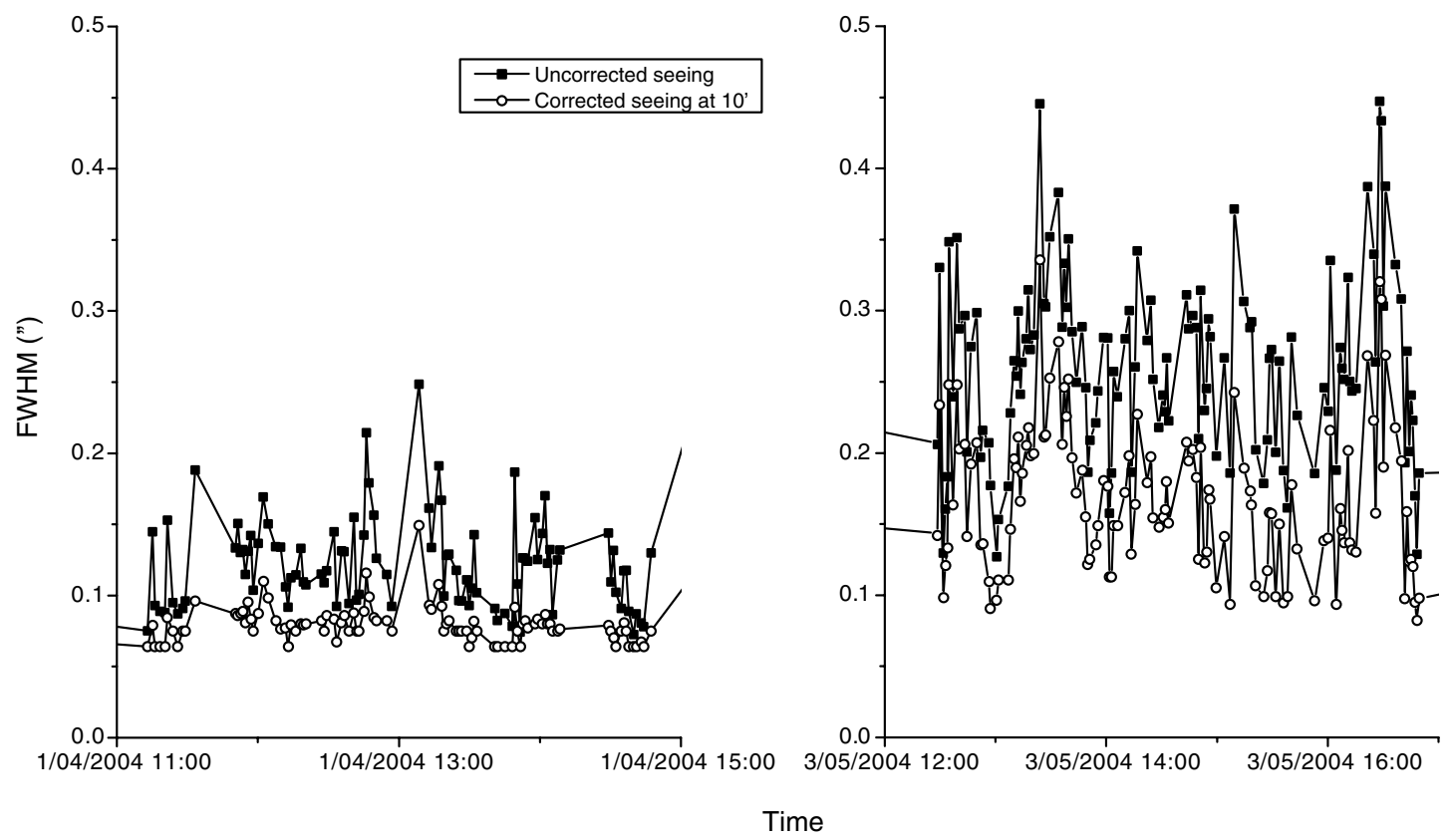

FIG. 8. - Time series of both natural and corrected seeing in the $V$-band for 2004 April 1 (left) and May 3 (right), for a tower-mounted $2.4 \mathrm{~m}$ Dome C telescope with a GLAO system, showing the range of performance that such a system could be expected to achieve. 
of correction brings the seeing almost always below $0.1^{\prime \prime}$. On this day, the free atmosphere was very stable and the variations of turbulence in the boundary layer were well compensated by the system. On May 3, however, the level of correction is more variable. The natural seeing, this time dominated by a turbulence layer in the troposphere, is poorer and oscillates rapidly between $0.1^{\prime \prime}$ and $0.4^{\prime \prime}$. In such conditions, the corrected resolution is equally variable. The GLAO system is insensitive to the troposphere turbulence, and while about $0.1^{\prime \prime}$ is still systematically removed, the seeing variations are still large.

\section{CONCLUSION}

The full potential of the unique atmospheric characteristics at Dome $\mathrm{C}$ station on the Antarctic plateau will not be realized until very large-aperture facilities, such as the $8.4 \mathrm{~m} \mathrm{LAPCAT}$ telescope (Storey et al. 2006) or the $22 \mathrm{~m}$ Antarctic Giant Magellan Telescope (Angel et al. 2004), with sophisticated instrumentation suites, are deployed there. The necessary next step toward such future facilities is to develop and deploy smaller-scale optical/infrared telescopes such as the $2.4 \mathrm{~m}$ PILOT (Pathfinder for an International Large Optical Telescope) (Saunders et al. 2008).

One of the key design issues for this first generation of $2 \mathrm{~m}$ class telescopes is to determine the best method to use in order to overcome the turbulent boundary layer at the Dome $\mathrm{C}$ site. The two main options are to mount the telescope on a $\sim 30 \mathrm{~m}$ tower with a fast tip-tilt guiding system, or to install the telescope at ground-level with a ground-layer adaptive optics system. The key purpose of this article has been to examine the relative performance of these two options. We have also extended the analysis by looking at the performance of a tower-mounted telescope with a GLAO system.

There are clearly different niches in parameter space in terms of achieved resolution, corrected FOV, and operating wavelength range for the three options investigated here. The widest FOV (potentially up to $40^{\prime}$ ) can be achieved with a towermounted telescope. This should achieve a resolution of $\sim 0.3^{\prime \prime}$ across this whole field for wavelengths from the visible $(0.7 \mu \mathrm{m})$ to the near-infrared $(2.5 \mu \mathrm{m})$. The highest level of correction can be achieved with a tower-mounted telescope equipped with a GLAO system. In this mode, close to the diffraction limit in $I$ band ( 0.1" FWHM) should be achieved, and the free-atmosphere seeing should be improved by at least a factor of 2 in $V$ band (to obtain $0.10-0.15^{\prime \prime}$ FWHM) over relatively wide $\left(\sim 10^{\prime}\right)$ fields. A ground-level telescope equipped with a GLAO system should achieve a reasonable resolution $\left(0.1^{\prime \prime}-0.2^{\prime \prime}\right)$ at wavelengths from $I$ band to $K$ band but is limited to much smaller fields $\left(<4^{\prime}\right)$ than the other options. This might change, though, if more than three guide stars can be implemented, leading to a better GLAO performance for a given FOV, or a larger corrected FOV, for a given performance threshold. Our current results therefore are an indication of the minimal perfor- mance that can be reach from the ground, using the simplest possible GLAO system.

Apart from the performance comparison, there are a number of practical issues to be considered. For example, the towermounted telescope option requires onsite construction, requires heavy materials to be shipped in from the coast, provides a less accessible working environment for winter-over astronomers and support staff, may introduce difficulties associated with the higher wind speeds and greater temperature fluctuations at the telescope altitude, and will require a guide system to overcome residual tower wind-shake. However, the tower represents a useful piece of infrastructure that can be used as a platform for future and more ambitious projects, allows for further improvements like the addition of a high-order classical (NGS or LGS) AO system (Lawrence et al. 2008) or a lucky imaging system, and allows a simple humidity control system (Saunders et al. 2008) due to the nature of the temperature and humidity profile in the ground layer.

The ground-level telescope equipped with a GLAO system does not allow very high-resolution observations (e.g., with a lucky imaging system or a high-order high-contrast AO system), may be technologically challenging (if, for instance, it requires multiple high-power laser sources to work in a low temperature environment), and is more complicated optically. Advantages of the ground-based telescope are that it is easier to construct and to perform maintenance, the structure will experience lower wind speeds, the ambient temperature will be lower (representing a gain in thermal sensitivity), and that it is versatile (as shown in Travouillon et al. 2004, the same GLAO system would perform almost equally well at the South Pole).

Ultimately the design option chosen for this telescope should depend on the science drivers for the facility. Many science cases (see Lawrence et al. 2008, in preparation) have been identified (such as the search for distant high-redshift galaxies and supernovae, or the mapping of large-scale structure through weak gravitational lensing) that require very wide field imaging in the optical and/or the near-infrared with a stable highresolution (though not necessarily at the telescope diffraction limit); these cases drive the design toward the tower-mounted solution. Other science cases (such as population studies of nearby galaxies, and mapping the molecular phase of the Galaxy) rely on high sensitivity in the mid-infrared, or high spatial resolution in the near-infrared; these science cases drive the design toward the ground-based GLAO option.

This research was funded by the NSF Office of Polar Programs (OPP), the Australian Research Council (ARC), the ARENA EC FP6 (RICA-026150), and the Netherlands Research School of Astronomy (NOVA). We would like to thank the Institut Paul Emile Victor (IPEV) and PNRA for the logistical support. We are also grateful to the referee who helped considerably to improve the clarity of this article. 


\section{REFERENCES}

Agabi, A., et al. 2006, PASP, 118, 344

Angel, J. R. P., Lawrence, J. S., \& Storey, J. W. V. 2004, Proc. SPIE, 5382, 76

Burton, M. G., Lawrence, J., Ashley, M., et al. 2005, PASA, 22, 199

Fried, D. 1966, J. Opt. Soc. Am., 56, 1372

Gillingham, P. R. 1991, Proc. Astron. Soc. Australia, 9, 55

Hagelin, S., Masciadri, E., Lascaux, F., \& Stoesz, J. 2008, MNRAS, 387,1499

Hammerschlag, R. H., Bettonvil, F. C. M., \& Jaegers, A. P. L. 2006, Proc. SPIE, 6273, 627310

Jolissaint, L. 2008, Journal of the European Optical Society, submitted

Jolissaint, L., Veran, J. P., \& Conan, R. 2006, J. Opt. Soc. Am. A, 23,382

Lawrence, J. S. 2004, PASP, 116, 482

Lawrence, J. S., Ashley, M. C. B., Jolissaint, L., Storey, J. W. V., \& Travouillon 2008, A\&A, 120, 1119

Lawrence, J. S., Ashley, M. C. B., Kenyon, S., Storey, J. W. V., Tokovinin, A., Lloyd, J. P., \& Swain, M. 2004b, Proc SPIE, 5489,174

Lawrence, J. S., Ashley, M. C. B., Tokovinin, A., \& Travouillon, T. 2004a, Nature, 431, 278

Le Roux, B., Carbillet, M., Langlois, M., Trinquet, H., Burgarella, D., Ferrari, M., \& Schmider, F-X. 2008, Proc. SPIE, 7015, 70154G

Marks, R. D., Vernin, J., Azouit, M., Manigault, J. F., \& Clevelin, C. 1999, A\&AS, 134, 161

Murtagh, F., \& Sarazin, M. 1993, PASP, 105, 932
National Astronomical Observatory of Japan 2003, Subaru telescope seeing (http://www.naoj.org/Observing/Telescope/ImageQuality/ Seeing/)

Rigaut, F. 2001, ESO Conf. Workshop Proc. 58, in Beyond Conventional Adaptive Optics, E. Vernet, R. Ragazzoni, S. Esposito, \& N. Hubin, 11

Roddier, F., Cowie, L., Graves, J. E., Songaila, A., \& McKenna, D. 1990, Proc. SPIE, 1236, 485

Saunders, W., Gillingham, P., McGrath, A., Haynes, R., Brzeski, J., Storey, J., \& Lawrence, J. 2008, Proc. SPIE, 7012, 70124F

Schroeder, D. J. 2000, Astronomical Optics, (Amsterdam, Boston: Academic Press), 2nd ed.

Storey, J. W. V., Angel, J. R. P., Lawrence, J. S., Hinz, P. M., Ashley, M. C. B., \& Burton, M. G. 2006, Proc. SPIE, 6267, 62671E

Tatarski, V. I 1961, Wave Propagation in a Turbulent Medium, (New York: McGraw-Hill)

Tokovinin, A. 2004, PASP, 116, 941

Travouillon, T., Ashley, M. C. B., Burton, M. G., Storey, J. W. V., \& Loewenstein, R. F. 2003, A\&A, 400, 1163

Travouillon, T., Lawrence, J. S., \& Jolissaint, L. 2004, Proc. SPIE, 5490, 934

Trinquet, H., Agabi, A., Vernin, J., Azouit, M., Aristidi, E., \& Fossat, E. 2008, PASP, 120, 203

Vernin, J., \& Muñoz-Tuñóz, C. 1992, A\&A, 257, 811

Walden, V. P., Town, M. S., Halter, B., Storey, J. W. V. 2005, PASP, 117,300

Ziad, A., Aristidi, E., Agabi, A., Borgnino, J., Martin, F., \& Fossat, E. 2008, A\&A, in press 